\title{
Perfil epidemiológico general de las quejas médicas atendidas en la Comisión Nacional de Arbitraje Médico. 1996-2007
}

Germán E Fajardo-Dolci, M Esp,( ${ }^{(1)}$ Francisco Hernández-Torres, Dr en C Sal, ${ }^{(1)}$ Javier Santacruz-Varela, MSP,(I) Mauricio Hernández-Ávila, Dr en Epi, ${ }^{(2)}$ Pablo Kuri-Morales, M en S Socmed, ${ }^{(3)}$ Enrique Gómez-Bernal MC.(1)

Fajardo-Dolci GE, Hernández-Torres F, Santacruz-Varela J,
Hernández-Ávila M, Kuri-Morales P, Gómez-Bernal E.
Perfil epidemiológico general de las quejas médicas atendidas
en la Comisión Nacional de Arbitraje Médico. 1996-2007. Salud Publica Mex 2009;5 I: I 19-1 25.

\begin{abstract}
Resumen
Objetivo. Analizar las quejas médicas atendidas en un periodo de II años con el fin de formular recomendaciones para prevenirlas y mejorar la planeación para su atención. Material y métodos. Se estudiaron las quejas de la Comisión Nacional de Arbitraje Médico (Conamed) recibidas entre 1996 y 2007 y se utilizaron medidas de estadística descriptiva para conocer su perfil general en relación con las variables de tiempo, lugar y persona. También se determinó la frecuencia de mala práctica médica evidente, así como la gravedad del daño que producen. Resultados. La solicitud de atención de quejas se incrementó entre 1996 y 2007, y existió menor demanda de atención en julio y diciembre. Dos estados del país concentran $69.7 \%$ de las quejas y $74 \%$ proviene de instituciones públicas. El $58 \%$ son de mujeres y los grupos de 25 a 34, y de 65 y más años tienen el mayor porcentaje. En $27 \%$ de las quejas atendidas hay evidencia de mala práctica médica y ginecología y obstetricia presenta el mayor porcentaje de quejas, daño físico y gravedad del daño. Las quejas por tratamiento son casi cuatro veces más que las de diagnóstico. Conclusiones. Es necesario mejorar la calidad del diagnóstico y el tratamiento e identificar medidas efectivas para el costo que reduzcan la mala práctica médica y la gravedad del daño físico en los pacientes.
\end{abstract}

Palabras clave: queja médica; calidad de la atención; mala práctica médica; México
Fajardo-Dolci GE, Hernández-Torres F, Santacruz-Varela J, Hernández-Ávila M, Kuri-Morales P, Gómez-Bernal E. Epidemiological general profile of complaints treated at the National Medical Arbitration Commission. 1996-2007. Salud Publica Mex 2009;5 I:I 19-125.

\begin{abstract}
Objective. To analyze medical complaints over a period of II years, for making recommendations for prevention and improving planning for responding. Material and Methods. We studied the medical complaints at the National Medical Arbitration Commission (Conamed, per its acronym in Spanish) between 1996 and 2007 using descriptive statistics to identify their general profile in relation to the variables of time, place and person. We also studied the frequency of evident medical malpractice as well as the severity of the damage it produces. Results. The request for responses to medical complaints increased between 1996 and 2007 and there was less demand between July and December. Two states in the country account for $69.7 \%$ of the complaints and $74.0 \%$ are from public institutions. Fifty-eight percent come from women and the 25-34 and 65+ age groups have the highest percentage. We found in $27.0 \%$ of the complaints there is evidence of medical malpractice and obstetrics and gynecology have the highest percentage of complaints, physical harm and severity of injury. Complaints about treatment are nearly four times more than diagnosis. Conclusions. It is necessary to improve the quality of diagnosis and treatment and identify cost-effective measures to reduce medical malpractice and the severity of physical damage in patients.
\end{abstract}

Key words: medical complaints, quality of care, medical malpractice; Mexico

(I) Comisión Nacional de Arbitraje Médico, México, DF, México.

(2) Subsecretaría de Prevención y Promoción de la Salud, Secretaría de Salud, México, DF, México.

(3) Centro Nacional de Vigilancia Epidemiológica y Control de Enfermedades, Secretaría de Salud, México, DF, México.

Fecha de recibido: 9 de mayo de 2008 - Fecha de aprobado: 30 de octubre de 2008

Solicitud de sobretiros: Dr. Germán E. Fajardo Dolci. Comisión Nacional de Arbitraje Médico. Mitla 250, piso 10, Narvarte 03020, México DF, México. Correo electrónico: gfajardo@conamed.gob.mx 
$\mathrm{L}^{\mathrm{a}}$ a Comisión Nacional de Arbitraje Médico (Conamed) fue creada en 1996 como organismo desconcentrado de la Secretaría de Salud de México para contribuir a solucionar las quejas médicas mediante una vía no judicial. ${ }^{1}$

Entre junio de 1996 y diciembre de 2007 la Conamed atendió un total de 18443 quejas mediante procedimientos de conciliación y arbitraje. La queja es la unidad principal de estudio de la institución y de forma operativa se define como una "petición a través de la cual una persona física por su propio interés o en defensa del derecho de un tercero solicita la intervención de la Conamed para impugnar la negativa de servicio médico o la irregularidad en su prestación" ${ }^{2}{ }^{2}$

En algunos casos las quejas médicas son efectuadas por pacientes que han sufrido un daño derivado de la atención recibida, y en esos casos se trata de sujetos con un evento adverso, el cual se define como un "incidente de la atención sanitaria que produce daño al paciente" en el marco conceptual de la Clasificación Internacional para la Seguridad del Paciente propuesto por la OMS. ${ }^{3}$ En el contexto de ese marco conceptual, el término atención sanitaria debe entenderse como atención médica, la cual es precisada por la Norma Oficial Mexicana del Expediente Clínico como el "conjunto de servicios que se proporcionan al individuo con el fin de promover, proteger y restaurar la salud". ${ }^{4}$ De acuerdo con esta definición, la atención médica es una actividad compleja por la pluralidad de servicios y fines que persigue, además de que en ella participan médicos, personal técnico, personal auxiliar, e intervienen aspectos de organización y gestión de servicios, por lo que las quejas se pueden deber a falencias de cualquiera de esos elementos.

Las quejas médicas son indicadores negativos de la calidad de atención y debido a ello se les relaciona con mala práctica médica. ${ }^{5-7}$ Por analogía, las quejas médicas pueden analizarse de acuerdo con el enfoque sistémico propuesto por Avedis Donabedian para evaluar la calidad de la atención médica. ${ }^{8}$ Según ese enfoque, algunas quejas pueden asociarse con factores estructurales, y otras con el proceso de atención.

En México se ha descrito un perfil general de las quejas médicas para periodos cortos de tiempo, así como su relación con la mala práctica médica. ${ }^{9,10}$ A través de estudios de otros países se conocen las causas de quejas y reclamos de pacientes por mala práctica médica y eventos adversos. ${ }^{11-15}$

Por otra parte, se sabe que las quejas médicas no sólo ocasionan daño a los pacientes, sino que también afectan la salud del personal médico al producirle estrés y preocupación, ${ }^{16,17}$ además de que disminuyen su satisfacción en el trabajo e interfieren con su vida personal. ${ }^{18}$ Estudios sobre la reacción emocional de los médicos refieren que pasan por tres etapas: impacto inicial, conflicto y resolución del conflicto. ${ }^{19}$

Dado que las quejas médicas son un indicador negativo de la calidad de atención, su análisis es necesario y útil para formular recomendaciones, tanto para prevenirlas como para mejorar la atención médica.

\section{Material y métodos}

La Conamed utiliza dos esquemas metodológicos complementarios para analizar y atender las quejas de los pacientes:

- Método individual. Basado en la opinión de los expertos.

- Método colectivo. Basado en el análisis epidemiológico.

Se eligió este último método para analizar las quejas médicas atendidas por la Conamed en el periodo de junio de 1996 a diciembre de 2007. El objetivo del análisis es profundizar en el conocimiento del perfil de las quejas médicas hasta ahora conocido y generar recomendaciones para contribuir a su prevención y a la mejora de la calidad de la atención médica.

El análisis de las quejas se desarrolló en cuatro etapas. En la primera de ellas se definieron variables epidemiológicas, cuya definición operativa para el presente estudio se describe a continuación:

- $\quad$ Tiempo: es la ocurrencia mensual y anual de las quejas médicas.

- Lugar: es su distribución por estado, institución y especialidad.

- Persona: es su ocurrencia por sexo y grupo de edad.

Además se definieron otras variables de interés para el objetivo del estudio:

a) Motivo de la queja: es el tipo de atención con el que se relaciona la queja, que puede ser de diagnóstico, tratamiento médico, tratamiento quirúrgico, atención de parto y puerperio y auxiliares de diagnóstico y tratamiento.

b) Evidencia de mala práctica médica: es un juicio del personal que atiende la queja médica sobre la existencia o no de errores en la atención del paciente, basado en la evidencia científica y la lex artis o buena práctica médica.

c) Daño: es la consecuencia física para el paciente que, cuando existe, puede ser con discapacidad temporal, con discapacidad permanente o muerte. 
En la segunda etapa se recopilaron los datos de las variables mencionadas y se usaron como fuente primaria los datos capturados de manera electrónica en el Sistema de Atención de Quejas Médicas (Saqmed) de la Conamed. ${ }^{20}$

En la tercera etapa se procesaron los datos y se efectuaron cálculos estadísticos para estimar la probabilidad con la que ocurren las quejas en la población. Debido a que en 2002 y 2003 se efectuaron modificaciones al Saqmed, los datos para algunas de las variables mencionadas estuvieron disponibles sólo a partir de esos años. Los datos se procesaron mediante aplicaciones informáticas del Cristal Reports de Business Objects, Excel y Epiinfo. Se efectuaron análisis univariado y bivariado para algunos resultados, que se describen a continuación.

- Análisis univariado: se analizaron frecuencias simples y razones para las variables cualitativas. Se destacó la frecuencia de ocurrencia por grupos de edad y sexo.

- Análisis bivariado: se aplicaron pruebas de significancia estadística, y prueba de hipótesis para proporciones y de asociación mediante la prueba "ji cuadrada".

La cuarta etapa consistió en interpretar los resultados y formular algunas recomendaciones dirigidas a contribuir a mejorar la calidad de atención y a prevenir las quejas médicas.

\section{Resultados}

\section{Quejas en relación con la variable tiempo}

Comportamiento mensual. Debido a la disponibilidad de datos en el Saqmed, esta variable sólo se pudo estudiar de 2002 a 2007. Se encontró que en ese periodo las quejas aumentan de manera constante de enero a junio, y en julio descienden $44 \%$ en relación con el mes previo. En agosto se incrementan de manera marcada, forman un pico, a partir del cual descienden en forma progresiva hasta diciembre, en el que incluso se reciben menos quejas que en enero. En seguida se presentan los cuartiles 1 y 3 por medio de gráficas, así como la mediana, lo que permite observar el comportamiento mensual o "estacional" de las quejas (figura 1).

Comportamiento anual. Entre junio de 1996 y diciembre de 2007 se recibieron 18443 quejas y durante ese periodo se presentaron las siguientes fluctuaciones anuales en la demanda de atención. Al principio existe un incremento, seguido de un descenso y un nuevo incremento. En 1996 se recibieron en sólo siete meses

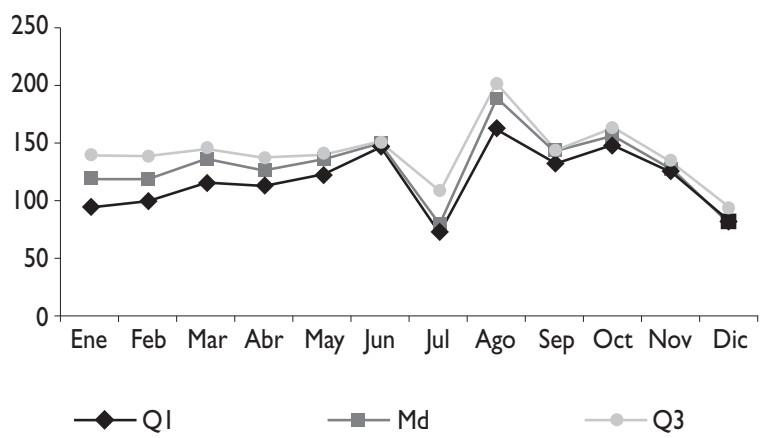

Fuente: Saqmed, Dirección General de Calidad e Informática. Conamed

Figura I. Comportamiento mensual de las Quejas ReClbidAs en Conamed, SEgún CUARTILES, 2002-2007

1158 quejas, y esa cifra se incrementó en forma constante hasta el 2000, en que se recibieron 1915 . A partir de 2001 se observa un descenso que se agudiza en 2002, con sólo 1172 quejas recibidas, y otra vez se incrementan hasta un máximo de 49\% en 2007, en que se reciben 1753 quejas. Se efectuó regresión lineal simple a los datos de este último periodo y se obtuvo un incremento promedio de 112 quejas por año.

\section{Quejas en relación con la variable lugar}

Por estado. De las 18443 quejas recibidas, 69.7\% corresponde a sólo dos entidades (Distrito Federal con 57.6\% y Estado de México con 12.1\%). Le siguen en orden descendente Guanajuato con $2.9 \%$, Veracruz con $2.1 \%$, Jalisco con $1.8 \%$ y Aguascalientes con 1.5 por ciento.

Por instituciones de salud. El 50\% de las quejas recibidas corresponde a usuarios del Instituto Mexicano del Seguro Social (IMSS), en segundo lugar a los servicios médicos privados con $26 \%$, en tercer lugar al Instituto de Seguridad y Servicios Sociales de los Trabajadores del Estado (ISSSTE) con 16\%, y en cuarto lugar a la Secretaría de Salud (población sin seguridad social) con 5 por ciento.

Evidencia de mala práctica en las quejas, según institución de salud. Por la limitación de datos ya expuesta, la evidencia de mala práctica (EMP) en las quejas según institución de salud se estudió en sólo 8750 quejas atendidas en el periodo 2002 a 2007 y de ese total únicamente se pudieron analizar $7259(83 \%)$ debido a que en 17\% restante faltaron datos en la documentación médica revisada por el personal de la Conamed. Del segmento estudiado, sólo 1959 presentaron evidencia de mala práctica, lo que corresponde a $27 \%$. La distribución 
por institución de estas quejas con EMP es como sigue: seguridad social $73 \%$, servicios médicos privados $22.6 \%$, sin seguridad social (SSA) 3.5\% y asistencia privada y social 0.9 por ciento.

El porcentaje de quejas con EMP en cada uno de los tipos de institución estudiados fue como sigue: seguridad social $26.2 \%$, servicios médicos privados $31.2 \%$, sin seguridad social (SSA) $22.3 \%$, asistencia privada $12.5 \%$ y asistencia social $43.5 \%$. Existe asociación estadísticamente significativa entre el tipo de institución médica y la frecuencia de $\operatorname{EMP}\left(\chi^{2}=42.65, p<0.0001\right)$. Por otra parte, cabe destacar que en unidades de servicios privados y de la asistencia privada se encontró el mayor porcentaje de quejas sin elementos por definir ( 39 y $36 \%$ ), y el menor porcentaje en las de la seguridad social (8\%), lo que podría reflejar de forma indirecta la calidad de la documentación médica en esas instituciones (figura 2).

Por especialidad. Las especialidades de las que más se reciben quejas en la Conamed son ginecología y obstetricia con $15 \%$, y en orden decreciente siguen las de ortopedia y traumatología con $12.5 \%$, urgencias médicas $10 \%$, cirugía general $7.3 \%$, y odontología $7 \%$. Estas cinco especialidades concentran $51.8 \%$ de las quejas recibidas. En cuanto a las especialidades no quirúrgicas, destacan las de medicina familiar con $6.3 \%$, y medicina interna con 2.4 por ciento.

Se efectuó regresión lineal simple para identificar la tendencia y se encontró que 12 especialidades presentan una tendencia clara de incremento o decremento anual promedio de quejas. En el primer caso se encuentran sobre todo los servicios de urgencias, cirugía general, traumatología y ortopedia, odontología y medicina familiar, con un incremento promedio respectivo de 42 , $38,15,12$ y 10 quejas por año. Por el contrario, destaca la cirugía neurológica con un decremento promedio de 36 quejas por año.

Por especialidad y gravedad del daño. En cuanto al análisis de la gravedad del daño en los pacientes, éste sólo se realizó en 1412 quejas con EMP atendidas desde 2003, año a partir del cual se registra esa variable en la Conamed. Debido a que las quejas en las que existe evidencia de daño físico relacionado con la atención médica cumplen con el criterio de evento adverso establecido por la OMS, estos resultados permiten una aproximación general indirecta al conocimiento de este tipo de eventos.

El 79.5\% de las quejas con EMP se concentra en 10 especialidades (figura 3) y, dentro de ellas, las cinco en las que se presenta el mayor porcentaje de daño físico fueron: ginecología y obstetricia con $69 \%$, pediatría con $64 \%$, oftalmología con $56 \%$, cirugía general con $55 \%$, y medicina familiar con $53 \%$. Si se analiza la mayor grave-

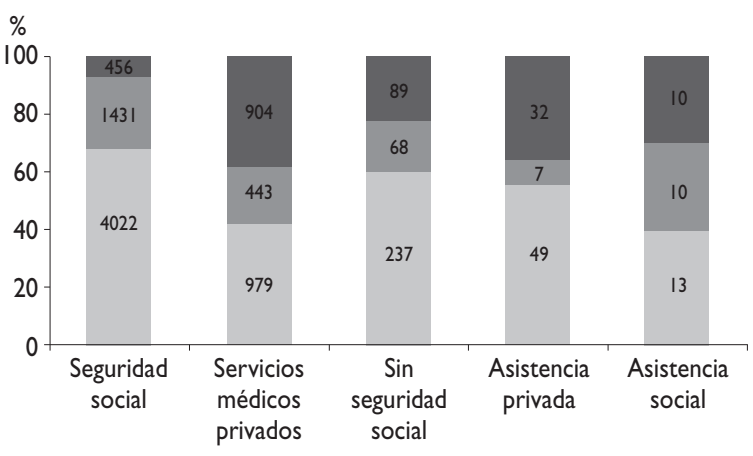

Sin elementos para definir

Con evidencia de mala práctica

Sin evidencia de mala práctica

Fuente: Saqmed, Dirección General de Calidad e Informática. Comisión Nacional de Arbitraje Médico. México

Figura 2. Quejas en Conamed, Según grupo institucioNAL Y EVIDENCIA DE MALA PRÁCTICA, 2002-2007

dad del daño por especialidad (sólo daño permanente y muerte), ese orden se modifica: ginecología y obstetricia ocupa el primer lugar con $53 \%$, seguida por pediatría con $42 \%$, oftalmología $40 \%$, medicina familiar $33 \%$ y traumatología y ortopedia 32 por ciento.

\section{Quejas en relación con la variable persona}

Según sexo. Las quejas presentadas en la Conamed son más frecuentes en el sexo femenino, ya que de 18443 quejas recibidas, 10705 correspondieron a mujeres (58\%) y 7738 a los varones (42\%). La tasa de quejas por población general fue de dos por 100000 habitantes en el año 2000, calculada a partir de las quejas en ese año y los datos del XII Censo Nacional de Población y Vivienda.

Según grupo de edad y sexo. La razón hombre-mujer por todos los grupos de edad es 0.7:1.0, pero presenta variaciones a lo largo de la vida, y asíse observan diferencias en tres momentos: a) en menores de un año, de 1 a 4 y de 5 a 14, la frecuencia es mayor en varones que en mujeres, con una superioridad de 28,26 y $18 \%$, respectivamente; b) en los siguientes cinco grupos de edad, las quejas son más frecuentes en mujeres que en varones, con una superioridad que oscila entre $40 \%$ para el grupo de 50 a 54 y $128 \%$ para el de 25 a $34 ;$ c) por último, a partir de los 55 años de edad, la frecuencia es similar en ambos sexos. Estas diferencias son estadísticamente significativas $\left(\chi^{2}=\right.$ 482.12, $p<0.0001$ ).

El porcentaje de quejas en relación con el total también varía por grupo de edad, ya que es menor en 


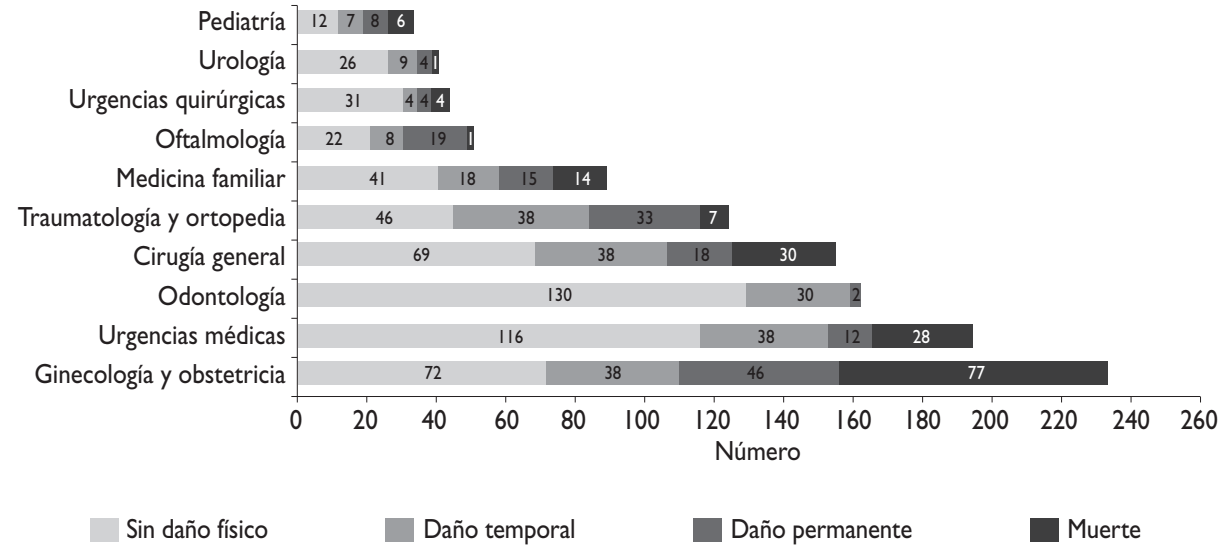

Fuente: Saqmed, Dirección General de Calidad e Informática. Comisión Nacional de Arbitraje Médico. México

Figura 3. Diez especialidades con mayor EVIdencia de mala práctica, según GRAVedad del daño, OCtUBRE 2003-2007

el de 1 a 4 años con $2.8 \%$ del total y mayor en el de 35 a 44 años con $19.4 \%$. Para ambos sexos se observa un comportamiento bimodal, con un pico en el grupo de 25 a 34 y otro en el de 65 y más años, es decir, predominan en adultos y personas de mayor edad, como se muestra en la figura 4.

\section{Quejas según motivo}

En el presente estudio se utilizó la clasificación de motivos de queja definida por la Conamed, a saber: entrevista inicial, diagnóstico, tratamiento, procedimientos auxiliares, relación médico-paciente y atención del parto y puerperio inmediato.

Las quejas relacionadas con el tratamiento quirúrgico ocuparon el primer lugar con $37.5 \%$, seguidas por tratamiento médico con $31.1 \%$, diagnóstico $18.2 \%$, atención de parto y puerperio $5.2 \%$, servicios auxiliares de diagnóstico y tratamiento $2.1 \%$, relación médicopaciente $1.7 \%$ y otros motivos 4.2 por ciento (figura 5).

\section{Discusión}

Desde la publicación del reporte del Instituto de Medicina de Estados Unidos (IOM) sobre el error humano en $1999,{ }^{21}$ se ha generado una basta literatura sobre la seguridad del paciente, eventos adversos y error médico; sin embargo, las publicaciones específicas sobre las quejas médicas son más limitadas y a veces sólo se las menciona indirectamente. $22-26$ De igual manera, en la literatura es común encontrar opiniones de médicos y

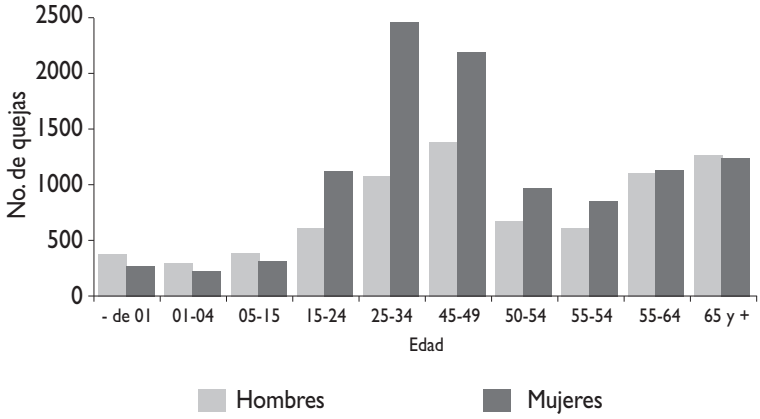

Fuente: Saqmed, Dirección General de Calidad e Informática. Comisión Nacional de Arbitraje Médico. México

Figura 4. Distribución de QUEJA POR SEXO Y GRUPO DE EDAD. ConAmed. 1996-2007

pacientes sobre la calidad de atención y los errores en medicina, pero son menos comunes las opiniones acerca de la queja médica. ${ }^{27-29}$

Este estudio analiza de manera simultánea las variables epidemiológicas básicas de tiempo, lugar y persona de las quejas médicas atendidas en poco más de una década en una institución especializada del sector salud, lo que ayuda a conocer con mayor claridad el perfil general de las quejas que se atienden en el país.

Con respecto a la variable tiempo, se encontró que la demanda de atención de quejas en general muestra una tendencia creciente, con promedio de 112 quejas 


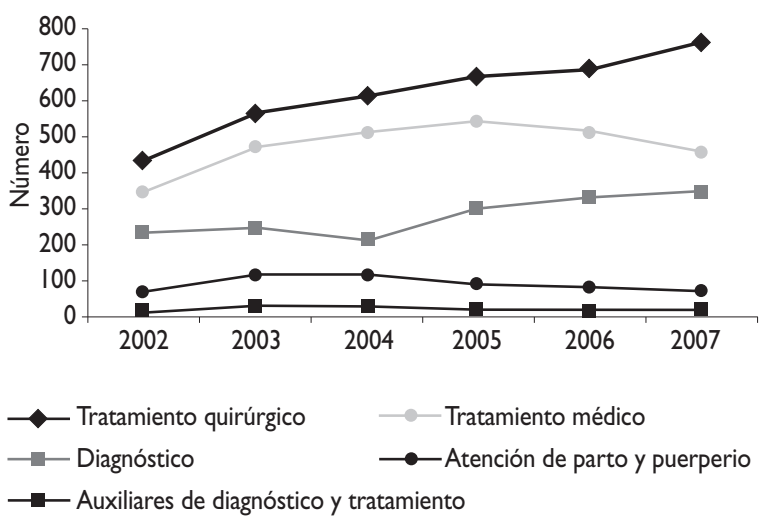

Fuente: Saqmed, Dirección General de Calidad e Informática. Comisión Nacional de Arbitraje Médico. México

Figura 5. Frecuencia de Quejas según motivo y con TENDENCIA MÁS PRONUNCIADA EN QUEJAS ATENDIDAS EN ConAmed, 2002- 2007

más por año en relación con el año previo, la cual podría estar asociada con las campañas de difusión por medios de comunicación masiva de la Conamed, así como por una mayor exigencia y expectativas de los pacientes hacia el personal médico y la calidad de atención, como lo refieren algunos estudios realizados en México y en otros países. ${ }^{30-33}$

El comportamiento mensual de la demanda de atención de quejas observado en este estudio sigue un curso similar a la capacidad de oferta de servicios de la Conamed, la cual varía en función de los periodos vacacionales de su personal, hecho que también puede influir en la demanda de servicios por la población.

Si bien la demanda de atención de quejas se ha incrementado, la tasa de dos por 100000 habitantes encontrada en este estudio es muy baja en comparación con la tasa de eventos adversos y de errores médicos, ${ }^{34-36}$ aunque se debe aclarar que no obstante que son acontecimientos relacionados con la calidad de atención, se trata de fenómenos diferentes y, por lo tanto, no comparables.

En relación con la variable lugar, los resultados muestran que $66 \%$ de las quejas correspondió a pacientes atendidos en instituciones de seguridad social (50\% del IMSS y $16 \%$ del ISSSTE), y $26 \%$ a pacientes atendidos en el sector privado; estos porcentajes son similares a la proporción de la población general que de forma regular se atiende en tales instituciones. Llama la atención que sólo 5\% de las quejas son de pacientes atendidos en unidades de la Secretaría de Salud, a las que de manera regular acude alrededor de un tercio de la población general. La baja demanda de atención de quejas de la población que acude a estas unidades, por lo general de escasos recursos económicos, podría deberse a factores de tipo sociocultural ya analizados en México. ${ }^{26,37}$

En cuanto a las especialidades médicas señaladas en las quejas médicas, destacan aquellas que utilizan procedimientos quirúrgicos y requieren hospitalización, lo cual expone a los pacientes a un mayor grado de riesgo para sufrir errores de la atención médica, en comparación con aquellos sujetos que reciben tratamiento ambulatorio o no quirúrgico. En este sentido, se observa un comportamiento similar al de un estudio previo en el que se utilizaron quejas de la Conamed, aunque de un periodo de tiempo menor. ${ }^{9}$

Los resultados relacionados con el sexo y grupos de edad indican que en general las quejas de pacientes de sexo femenino, recibidas en la Conamed, son más frecuentes que las recibidas de pacientes masculinos, ya que por cada 10 quejas de varones se presentan 14 de mujeres; sin embargo, esa conducta varía cuando se analiza por grupo de edad y llama la atención que desde los primeros años de la vida hasta los 14 años predominen las quejas del sexo masculino, entre los 25 y 34 años hay un marcado predominio del sexo femenino y en los grupos de mayor edad la frecuencia es similar. No se dispone de una explicación para esta conducta, pero en apariencia no se relaciona con la demanda de consulta ni con la distribución real de la población o pirámide poblacional, por lo que es muy probable que tales diferencias también obedezcan a factores socioeconómicos y culturales que deben estudiarse.

Los resultados acerca de los motivos de queja muestran que $73.6 \%$ se relaciona con el tratamiento, $20 \%$ con el diagnóstico y sólo 1.9\% con la relación médico-paciente. La poca consignación de quejas debidas a la relación médico-paciente ya ha sido referida en publicaciones previas y se atribuye a un sesgo de "medicalización" de quienes atienden las quejas. ${ }^{37}$ Este hecho debe impulsar la revisión de la clasificación y codificación de quejas que se emplea en la actualidad en el marco de clasificaciones de salud como la Clasificación Internacional de Enfermedades y la Clasificación Internacional para la Seguridad del Paciente, que ya se mencionaron.

Para finalizar, es necesario investigar otros factores socioculturales para ampliar la caracterización epidemiológica de las quejas médicas que permita explicar las diferencias encontradas. 


\section{Referencias}

I. Diario Oficial de la Federación. Decreto de creación de la Comisión Nacional de Arbitraje Médico (Conamed): México. Diario Oficial de la Federación del 03 de junio, 1996.

2. Diario Oficial de la Federación. Reglamento de Procedimientos para la Atención de Quejas Médicas y Gestión Pericial de la Conamed: México. Diario Oficial de la Federación del 25 de julio, 2006.

3. World Health Organization. The conceptual framework for the international classification for patient safety. Geneva:WHO 2007.Available from: http//www.who.int/patientsafety/taxonomy/icps_form/en/index.html 4. Secretaría de Salud. Norma Oficial Mexicana NOM-I68-SSAI-I 998. México: SSA, 1998.

5. Hickson BG, Federspiel FCH, Pichert WJ, Miller SC, Gauld-Jaeger J, Bost P. Patient complaints and malpractice risk. JAMA 2002;287:295I-2957.

6. Daniel AE, Burn RJ, Horarik S. Complaints about medical practice. Med J Aust 1999; 170:598-602.

7. Sage MW. Putting the patient in patient safety. Linking patient complaints and malpractice. JAMA 2002;287:3003-3005.

8. Donabedian A. Evaluating the quality of medical care. Milbank Mem Fund Q 1966;44:166-206.

9. Jimenez-Corona ME, Ponce-de-Leon RS, Rangel FS, Mohar BA. Epidemiology of medical complaints in Mexico: identifying a general profile. Int J Qual Health Care 2006; 18:220-223.

10. Tena-Tamayo C, Sotelo J. Malpractice in Mexico: arbitration no litigation. Br Med J 2005;33I:448-45I.

II. Fenn P, Diacon S, Gray A, Hodges R, Rickman N. Current cost of medical negligence in NHS hospitals: analysis of claims. $\mathrm{Br}$ Med J 2000;320:1567-|57|.

12. Bovjerg RR, Petronis RK. The relationship between physicians' malpractice claims history and later claims. Does the past predict the futur? JAMA 1994;272:142I-1426.

13. Hampton T. Payments for adverse events. JAMA 2006;296:1958. 14. Kravitz LR, Rolph EJ, McGuigan K. Malpractice claims data as a quality improvement tool. I. Epidemiology of error in four specialities. JAMA 1991;266:2087-2092.

15. Vincent AC, Coulter A. Patient safety: what about the patient? Qual Saf Health Care 2002; I I:76-80.

16. Aasland GO, Ferde R. Impact of feeling responsible for adverse events on doctors' personal and professional lives: the importance of being open to criticism from colleagues. Qual Saf Health Care 2005; 14:13-17.

17. Kessler PD, Sage MW, Becker JD. Impact of malpractice reforms on a supply of physician services. JAMA 2005;293:2618-2625.

18. Cooper GL, Rout U, Faragher B. Mental health, job satisfaction and job stress among general practitioners. Br Med J 1989;298:366-370.

19. Jain A, Ogden J. General practitioners' experiences of patients complaints: qualitative study. Br Med J 1999;3 18:I596-1599.
20. Sistema de Atención de Quejas Médicas (SAQMED). Comisión Nacional de Arbitraje Médico (Conamed). Serie histórica de datos 19962006.

21. Kohn LT, Corrigan JM, Donaldson MS (eds.). To err is human: building a safer health system. Washington, DC: National Academy Press 2000. 22. Graham KG, Itagaky WM,Volpintesta E, Hickson BG, Pichert WJ, Miller SC, et al. Patient complaints and risk of being sued. JAMA 2002;288:15851586 .

23. Armitage JK, Schneiderman JL, Bass AR. Response of physicians to medical complaints in men and woman. JAMA 1979;241:2186-2187. 24. Vincent $C$. Understanding and responding to adverse events. N Engl J Med 2003;348: 105I-I056.

25. Fisseni G, Pentzek M, Abholz HH. Responding to serious medical error in general practice --consequences for the GPs involved: analysis of 75 cases from Germany. Fam Pract 2008;25:9-13.

26. RaydelVS, Molina LJ, Solís TC. Aprender de lo sucedido.Análisis de las quejas presentadas ante la Comisión Nacional de Arbitraje Médico. Salud Publica Mex 200I;43:444-454.

27. Robinson AR, Hohmann KB, Rifkin JI, Topp D, Gilroy CM, Pickard JA, et al. Physician and public opinions on quality of health care and the problem of medical errors. Arch Intern Med 2002;162:2186-2190.

28. Bismark MM et al. Relationship between complaints and quality of care in New Zealand: a descriptive analysis of complaints and non-complaints following adverse events. Qual Saf Health Care 2006;15:17-22.

29. Fernandez-Varela MH.The National Medical Arbitration Commission and the quality of care. Gac Med Mex 1997;133:57-60.

30. Peña VR, Rodríguez MJR, López CJM. Factores asociados con la gestión de quejas contra médicos que laboran en hospitales del Instituto Mexicano del Seguro Social. Salud Publica Mex 2004; 46:210-2I5.

3I.Tena TC, Ruelas BJM, Sánchez GAE, Rivera CAE, Moctezuma B, Manuel LGR. Derechos de los pacientes en México. Rev Med Inst Mex Seguro Soc 2002;40:523-529.

32. Rees LJ. Patients views on quality care in general practice: Literature review. Soc Sci Med 1994;39:655-670.

33. Kravitz RL, Callahan EJ, Paternini D, Antonius D, Dunham M, Lewis CE. Prevalence and sources of patients' unmet expectations for care.Ann Intern Med 1996; I 25:730-737.

34. Leape LL et al.The nature of adverse events in hospitalized patients. Results of the Harvard Medical Practice Study II. N Engl J Med 1991;324:377-384.

35. Vincent $\mathrm{Ch}$ et al.Adverse events in British hospitals: preliminary retrospective record review. Br Med J 200I;322:5 17-5I9.

36. Weingart $\mathrm{SN}$ et al. Epidemiology of medical error. $\mathrm{Br}$ Med J 2000;320:774-777.

37. Infante CC. Quejas médicas. México: Editores de Textos Mexicanos, 2006. 